Rev. Adm. Saúde (On-line), São Paulo, v. 19, n. 74, jan. - mar. 2019, Epub 05 fev. 2019 http://dx.doi.org/10.23973/ras.74.153

ARTIGO ORIGINAL

\title{
Proposta de metodologia para ações de qualidade de vida no trabalho em serviços de saúde
}

Proposal of a methodology for actions in the area of quality of life at work in health services

\section{Milton M. Osaki ${ }^{1}$, Marcelo Pustiglione ${ }^{2}$}

1. Médico, administrador hospitalar e de sistemas de saúde. Médico da Secretaria da Saúde do Estado de São Paulo. São Paulo SP.

2. Médico do trabalho. Docente da Disciplina de Medicina do Trabalho do Instituto Oscar Freire da Faculdade de Medicina da Universidade de São Paulo. São Paulo SP

\section{RESUMO}

Introdução: A gestão de pessoas deve desenvolver ações que possibilitem às organizações a plena consecução de suas missões, neste caminho, a promoção do bem-estar e a busca da satisfação dos profissionais, através de práticas que contemplem a diversidade de suas necessidades, devem ser consideradas como fundamentais. Aceita-se que a valorização humana e consequente qualidade de vida no trabalho permitam a obtenção de resultados significativos para a organização. Qualidade de vida no trabalho: Em um conceito estrito, envolve a manutenção e preservação da saúde física, incluindo a garantia de condições saudáveis e seguras de trabalho, mas em um conceito mais amplo (qualidade final de um serviço prestado) envolve outros fatores relacionados aos recursos da organização e a inserção social do profissional. Equação da qualidade final: A gestão da qualidade do produto final pode ser representada por uma equação onde a qualidade final dependerá dos fatores: 1) qualidade dos recursos disponíveis para prestadores e usuários; 2) qualidade técnico-operacional, isto é, a forma mais adequada de executar o processo de trabalho; 3 ) qualidade de vida no trabalho, ou seja, a disponibilização ao trabalhador de condições saudáveis e seguras de trabalho; e 4) qualidade de vida geral, aquela ofertada ao conjunto da população da qual 
o trabalhador também faz parte. Propostas de ações: 1) qualificação do ambiente de trabalho (conforto, instalações e equipamentos ergonômicos); 2) evitar situações estressoras ocupacionais relacionadas à jornada, repressão à iniciativa profissional, má comunicação entre níveis hierárquicos, falta de perspectiva de ascensão pessoal, etc.; 3) controle dos riscos ocupacionais conforme e aderente as normas regulamentadoras oficiais da área; 4) promoção, prevenção e proteção às doenças e anomalias clínicas comuns como obesidade, tabagismo, alcoolismo, diabetes e hipertensão arterial, junto a promoção de hábitos saudáveis como atividade física, lazer, alimentação correta; 5) oferta de programas de educação financeira, preparação para aposentadoria, envelhecimento saudável, apoio à mãe trabalhadora, etc.

Discussão e Conclusão: Cabe ao serviço de saúde ocupacional da organização desenvolver os programas de qualidade de vida no trabalho e qualidade de vida geral, monitorar indicadores de resultados como índice de absenteísmo e taxa de acidente de trabalho, promover a melhoria contínua utilizando-se de ferramentas como o ciclo PDCA, e promover parcerias com as comissões de trabalhadores e de infecção hospitalar. A conscientização tanto do trabalhador como do gestor para o desenvolvimento da cultura prevencionista é fundamental para a satisfação do profissional e melhoria da qualidade no atendimento do usuário do serviço de saúde.

Palavras-chave: Qualidade de Vida; Gestão de Pessoas; Saúde Ocupacional; Serviços de Saúde

\section{ABSTRACT}

Introduction: People management must develop actions that enable organizations to fully achieve their missions, in this way, promoting well-being and seeking the satisfaction of professionals, through practices that contemplate the diversity of their needs, should be considered as fundamental. It is accepted that human valorization and consequent quality of life at work allow the achievement of significant results for the organization. Quality of life at work: In a strict concept, it involves the maintenance and preservation of physical health, including the guarantee of healthy and safe working conditions, but in a broader concept (final quality of a service provided) involves other factors related to resources of the organization and the social insertion of the professional. Final quality equation: Quality management of the final product can be represented by an equation where the final quality will depend on the factors: 1) quality of the resources available to suppliers and users; 2) technicaloperational quality, that is, the most adequate way of performing the work process; 3) quality of life at work, that is, the availability to the worker of healthy and safe working conditions; and 4) general quality of life, that offered to the whole population of which the worker is also a part. Proposals for actions: 1) qualification of the working environment (comfort, facilities and ergonomic equipment); 2) avoiding occupational stressors related to the journey, repression of professional initiative, poor communication between hierarchical levels, lack of perspective of personal ascension, etc.; 3) control of occupational risks according to and adhering to the official regulatory standards of the area; 
4) promotion, prevention and protection of diseases and common clinical abnormalities such as obesity, smoking, alcoholism, diabetes and hypertension, along with the promotion of healthy habits such as physical activity, leisure, proper nutrition; 5) offer financial education programs, preparation for retirement, healthy aging, support to the working mother, etc. Discussion and conclusion: It is up to the organization's occupational health service to develop quality of life programs at work and general quality of life, monitor outcome indicators such as absenteeism index and work accident rate, promote continuous improvement using tools such as the cycle PDCA, and promote partnerships with employee committees and hospital infection. The awareness of both the worker and the manager for the development of the prevention culture is fundamental for the satisfaction of the professional and improvement of the quality in the care of the user of the health service.

Keywords: Quality of Life; People management; Occupational Health; Health services.

\section{INTRODUÇÃO}

A administração cientifica, surgida no século XX, tem papel importante na ajuda às instituições para que estas possam operar de maneira exitosa no atual ambiente globalizado e competitivo. As estratégias administrativas são um conjunto de decisões e ações que determinam o desempenho e proporcionam resultados para uma corporação. A gestão de pessoas delineou-se como fundamento estratégico a partir da década de 1980, quando se evidenciou que a administração dos recursos humanos permitia a realização das estratégias organizacionais com consequente obtenção de diferencial competitivo. (1)

Anteriormente, gestão de pessoas era entendida como uma área estritamente profissional e administrativa, limitando-se à execução de atividades rotineiras (1). Segundo Becker, Ulrich e Huselid, (1) apenas na década de 1980 a questão da estratégia de negócios passou a ser considerada, iniciando-se 0 processo de reconhecimento da importância da gestão de pessoas.

Atualmente, a gestão de pessoas deve desenvolver ações que possibilitem às organizações a plena consecução da sua visão, missão, objetivos estratégicos e valores organizacionais.

Neste trajeto, a promoção do bem-estar e a busca da satisfação das pessoas, através da execução de políticas e práticas que contemplem a diversidade de seus anseios e necessidades, devem ser consideradas como pilares fundamentais. Aceita-se que a valorização humana e consequente qualidade 
de vida no trabalho permitem a obtenção de resultados significativos para a organização. (2)

A discussão sobre o tema qualidade de vida no trabalho tornou-se assunto atual inserido dentro de um processo, não apenas de busca do desenvolvimento humano, mas também organizacional.

\section{A QUALIDADE DE VIDA NO TRABALHO}

A Organização Mundial da Saúde (OMS) conceitua a qualidade de vida como "a percepção do indivíduo de sua posição na vida, no contexto da cultura e sistema de valores nos quais ele vive e em relação aos seus objetivos, expectativas, padrões e preocupações". Trata-se de um conceito amplo no qual aspectos variados da vida estão envolvidos de forma complexa, tais como saúde física, estado psicológico, nível de independência, crenças pessoais e suas relações com o meio em que vive (3).

Os estudos sobre qualidade de vida no trabalho (QVT) surgiram na década de 1950, na Inglaterra, com pesquisas de Eric Trist e colaboradores do Tavistock Institute, analisando a relação indivíduo-trabalho-organização. Estes cientistas estudaram a organização do trabalho, sob visão sócio técnica, embasados na satisfação do funcionário no trabalho (4).

Richard Walton, considerado pesquisador pioneiro para estabelecimento de metodologia de avaliação da QVT referiu na década de 1970 que "a expressão qualidade de vida tem sido usada com crescente frequência para descrever certos valores ambientais e humanos, negligenciados pelas sociedades industriais em favor do avanço tecnológico, da produtividade e do crescimento econômico". (5)

O modelo proposto por Walton contém oito variáveis a serem consideradas na avaliação da QVT: 1) compensação adequada e justa; 2) condições de segurança e saúde no trabalho; 3) oportunidade imediata para a utilização e desenvolvimento da capacidade humana; 4) oportunidade futura para crescimento contínuo e segurança; 5) integração social na organização de trabalho; 6) constitucionalismo na organização de trabalho; 7) o trabalho e o espaço total da vida; e 8) relevância social da vida no trabalho.

Eda Fernandes (1996) (6), pesquisadora brasileira pioneira no estudo em QVT, a define como a "gestão dinâmica e contingencial de fatores físicos, tecnológicos e sociopsicológicos que afetam a cultura e renovam os climas organizacionais, refletindo-se no bem-estar do trabalhador e na produtividade das empresas". Esta pesquisadora defende que a QVT pode ser entendida com uma estratégia, cuja "meta principal de tal abordagem volta-se para a conciliação dos interesses dos indivíduos e das organizações, ou seja, ao mesmo tempo em que melhora a satisfação do trabalhador, melhora a produtividade da empresa". Para esta autora o conceito de QVT englobaria, além de atos legislativos que protegem o trabalhador, o atendimento a 
necessidades e aspirações humanas calcadas na ideia de humanização do trabalho e na responsabilidade social da empresa.

Dantas (1996) (7) relaciona QVT com gestão pela qualidade total (GQT) com grande enfoque na promoção à saúde com controle das doenças crônicas relacionadas ou não ao ambiente do trabalho. Para este pesquisador, o monitoramento, controle de doenças como a dislipidemia, diabetes, hipertensão arterial e promoção à saúde no ambiente do trabalho proporcionam QVT.

França (1996) (8), França (1997) (9), Albuquerque e Limogi-França (1998) (10) e Limongi-França e Zaima (2002) (11) consideram a QVT “como o conjunto de ações de uma empresa que envolve a implantação de melhorias e inovações gerenciais e tecnológicas no ambiente de trabalho". Estes mesmos autores argumentam que "A construção da QVT ocorre a partir do momento em que se olha a empresa e as pessoas como um todo, o que chamamos de enfoque biopsicossocial". França (1997) considera que "O posicionamento biopsicossocial representa o fator diferencial para a realização de diagnósticos, campanhas, criação de serviços e implantação de projetos voltados para a preservação e desenvolvimento das pessoas durante o trabalho na empresa" e enfatiza a importância da ética da condição humana nesse contexto, afirmando que "Esta ética busca desde a identificação, eliminação, neutralização ou controle dos riscos ocupacionais observáveis no ambiente físico, padrões de relações de trabalho, carga física e mental requerida por cada atividade, implicações políticas e ideológicas, dinâmica da liderança empresarial e do poder formal até o significado do trabalho em si, relacionamento e satisfação no trabalho".

Para Chiavenato, (2002) (12) QVT "representa o grau em que os membros da organização são capazes de satisfazer suas necessidades pessoais através de sua atividade na organização"; e, que o conceito de QVT não deve estar restrito apenas à melhoria das condições de emprego, mas sim, ser amplo no sentido de que o próprio trabalho seja de qualidade, proporcionando a valorização à vida.

Desprende-se neste contexto o entendimento de que QVT envolve a manutenção e preservação da saúde física, incluindo a garantia de condições saudáveis e seguras de (e no) trabalho.

Claro que a qualidade do produto final (QPF), "um trabalho ou serviço de qualidade", missão e objetivo de toda e qualquer empresa, tem o seu lugar de destaque, mas não deve ser confundido, muito menos reduzido à qualidade de vida no trabalho, apenas porque, como dizem alguns autores ligados aos "círculos de controle de qualidade" propicia "sobrevivência, desenvolvimento e crescimento". As necessidades humanas vão além disso!

Esta situação se torna mais complexa quando o produto final não é um parafuso ou um automóvel, mas sim a satisfação, saúde ou resolução de um problema de uma pessoa ou grupo de pessoas. Aí, aspectos relacionados à facilitação de acesso, níveis de conforto físico, psíquico e social, além de apoio para solucionar necessidades declaradas ou observadas refletem diretamente 
na QPF e dependem, não raramente, mais do prestador do serviço do que de regras ou manuais de procedimento.

Visto desta maneira, integral, sistêmica e biopsicossocial, a gestão da QPF implica numa "relação dinâmica multifatorial" que pode ser representada por uma "equação da qualidade" que considera quatro membros qualitativos fundamentais, a saber:

1) a qualidade dos recursos disponíveis para prestadores e usuários que tem impacto direto na relação prestador-usuário;

2) a qualidade técnico-operacional, isto é, a forma mais adequada, eficaz e ética de executar as diferentes etapas do processo de trabalho;

3) a qualidade de vida no trabalho, ou seja, a disponibilização ao trabalhador de condições saudáveis e seguras de trabalho; e

4) a qualidade de vida geral, aquela ofertada ao conjunto da população da qual o trabalhador também faz parte.

\section{A EQUAÇÃO DA QUALIDADE}

A seguir são explicados os elementos qualitativos fundamentais da equação da qualidade.

\section{Qualidade dos recursos disponíveis (QRD)}

Os recursos organizacionais representam os meios que as instituições possuem para atingirem seus objetivos, não representados apenas e nem prioritariamente pelo dinheiro investido, mas também às matérias primas utilizadas no processo produtivo, nos serviços prestados, instalações, equipamentos e colaboradores. No mundo globalizado de hoje a garantia, continuidade e harmonia dos recursos organizacionais são fundamentais para possibilitar o bom desempenho dos colaboradores, obtido especialmente por meio de projetos de capacitação da força de trabalho, e, desta forma, sustentar a vantagem competitiva da organização.

Nesse contexto devem ser considerados:

a) Recursos físicos, ambientais ou materiais - Dizem respeito ao espaço físico, edificação, estrutura, sinalização, tecnologia, método, rotina e procedimentos que têm em vista a obtenção dos melhores resultados com o menor custo e a garantia da satisfação dos usuários e dos prestadores de serviço ou colaboradores. São medidas frequentemente simples, que facilitam a locomoção, orientação e conforto de todos, especialmente daqueles com limitações físicas, e que, além disso, humanizam e garantem segurança ao ambiente e ao processo de trabalho. Dois elementos devem ser salientados pelo impacto direto na 
QRD: 1) tecnológicos: ter disponibilizado e em condições de uso, sem limitações, o melhor recurso possível e indispensável para atingir os objetivos do serviço e responder com agilidade e eficiência as necessidades do usuário; e b) metodológicos: relacionados a como as tarefas serão realizadas num escopo de alto desempenho. Envolvem toda gama de recursos: humanos, materiais e físicos, tendo como objetivo não a questão técnica propriamente dita, mas sim oferecer ao usuário condições que zerem ou minimizem agentes de insatisfação preveníveis e controláveis, tais como não cumprimento de horários ou prazos, cancelamento de atendimento, desinformação, etc. que, somados à tensão geralmente presente no usuário que busca resolver um problema, certamente compromete o resultado final, além de deteriorar a relação usuário-prestador com grande impacto negativo na qualidade do ambiente.

b) Recursos financeiros - Representado pelo capital, fluxo de caixa, investimentos, etc. Uma boa gestão financeira deve priorizar e disponibilizar recursos para garantir a qualidade do produto ou do serviço prestado e a remuneração continuada e digna dos trabalhadores.

c) Recursos humanos - Trata-se da totalidade de colaboradores que compõe uma organização, independentemente do nível hierárquico. Neste aspecto devem ser considerados os aspectos qualitativos (formação, aptidão, capacitação, motivação, etc.) e quantitativo (número adequado e suficiente) de colaboradores. Por exemplo: trabalhador não capacitado e/ou sobrecarregado sofre no desempenho de sua atividade laboral.

d) Recursos administrativos e/ou organizacionais - São as atividades de planejamento, organização e controle das tomadas de decisão. Facilidade e universalização de acesso, disponibilidade dos recursos e desburocratização dos procedimentos são variáveis fundamentais intervenientes no nível de satisfação do usuário com reflexo imediato na melhoria da relação prestador-usuário. São pré-requisitos para atingir este nível de qualidade: 1) conhecimento perfeito de todo o processo de trabalho (desde a caracterização da clientela e do tipo da demanda até as questões técnicas envolvidas); 2) Adoção de visão abrangente, sistêmica e dinâmica do trabalho; e 3) Trabalho em equipe com compartilhamento da responsabilidade.

\section{Qualidade técnico-operacional (QTO)}

Este membro da equação está relacionado ao modelo de gestão adotado. A qualidade é garantida quando a forma utilizada está alinhada à missão da organização, mostra-se eficaz nas avaliações sistemáticas e é ética, moral e justa. Objetivos e processos de trabalho deve ser claro, de conhecimento de todos e fruto de construção participativa da qual participam representantes de todos os atores envolvidos. Definidos estes parâmetros, procedimentos 
operacionais padrão serão estruturados, implantados, avaliados e corrigidos sempre que necessário adotando-se o modelo de melhoria continua (PDCA). Tendo como base esta ferramenta administrativa, devem ser estruturados, implantados e implementados programas de capacitação, educação continuada e treinamento em serviço para o conjunto dos trabalhadores.

\section{Qualidade de vida no trabalho (QVT)}

Esta categoria da qualidade está intimamente associada à satisfação das necessidades humanas e fatores motivacionais e higiênicos.

a) Satisfação das necessidades humanas - $O$ modelo de hierarquia das necessidades humanas, conhecido como "Pirâmide de Maslow" (13), mostrado abaixo na Figura 1, até os dias de hoje caracteriza-se como excelente ferramenta de análise. Maslow (1943) salienta que a satisfação destas necessidades se configura como agente motivador a partir do qual o homem participa com prazer de suas atividades e tarefas, fato que certamente repercute positivamente na QPF.

b) Fatores motivacionais e higiênicos - De acordo com HERZBERG (14, 15) a motivação está alicerçada no ambiente externo e no trabalho do indivíduo. Existem fatores motivacionais ou satisfacientes (delegação de responsabilidade, liberdade de exercício, promoção ou plano de carreira, uso pleno de habilidades, etc.) que guardam relação positiva com a qualidade do desempenho e saúde mental, emocional. Existem também fatores higiênicos ou satisfacientes que quando situados em nível abaixo dos considerados adequados pelo trabalhador geram insatisfação (condições de trabalho e conforto, política da organização e administração, relação com a chefia, competência dos superiores, salário, segurança no cargo e relações interpessoais. Análise séria e intervenções adequadas visando satisfação progressiva das necessidades do trabalhador e direcionamento dos esforços no sentido de maximizar os fatores motivacionais e minimizar os higiênicos certamente representarão contribuição positiva para a melhoria da QVT. 


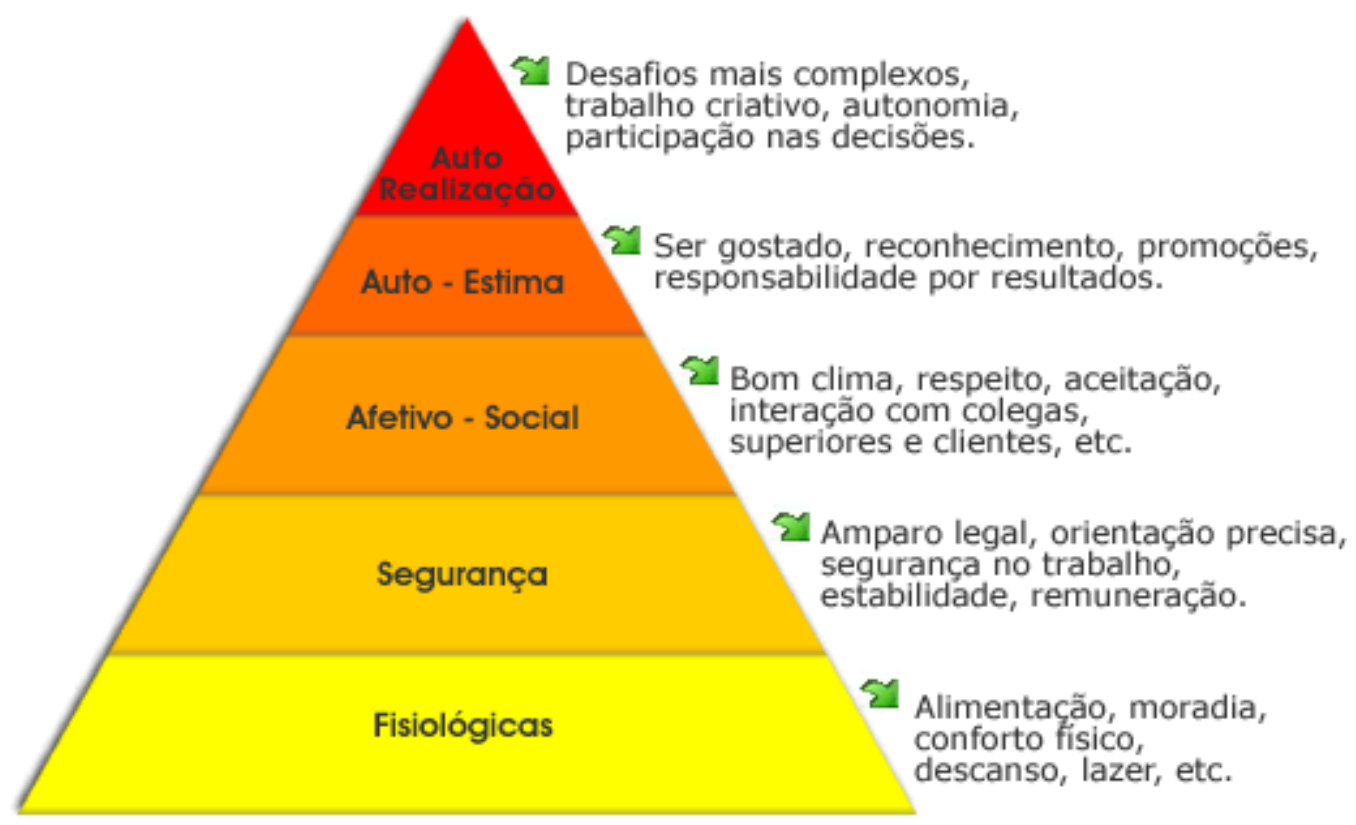

Figura 1. Pirâmide de Maslow.

\section{Qualidade de vida geral (QVG)}

Satisfação das necessidades e fatores motivacionais e higiênicos gerais ou cidadãos também tem importante representação na avaliação desta variável qualitativa.

Deve ser considerado que todos os elementos envolvidos na relação prestadorusuário carregam consigo realizações e/ou frustrações que, em determinado momento, por conflitantes ou semelhantes, podem interferir na relação, comprometendo o produto final.

Portanto, é fundamental análise e identificação destas questões para que sejam corrigidas ou minimizadas prioritariamente no prestador e idealmente no usuário. Assim, as empresas devem estar projetadas na comunidade atuando de forma globalizante como agente modificador de condições insalubres de qualquer natureza e neutralizador de fatores de risco.

Assim, a equação da qualidade pode ser expressa da seguinte forma:

$$
Q P F=Q R D+Q T O+Q V T+Q V G
$$

ou seja, a qualidade do produto ou do serviço produzido ou ofertado não é um elemento isolado e sim o resultado da somatória de vários elementos íntima e dinamicamente envolvidos e inter-relacionados. Em decorrência, a QVT deve ser encarada como parte desse modelo de gestão e não como um programa ou modismo.

Merece salientar que este modelo é complexo, pois envolve valores, representações, capacitação, conhecimento, desejos, ambições, enfim todo um conjunto de sentimentos e angústias individuais num contexto de relações 
interpessoais, seja com o conjunto de outros indivíduos envolvidos no processo produtivo, seja com o conjunto de clientes/usuários.

Neste contexto, o trabalho deve ser visto como um meio de desenvolvimento e realização e não apenas como um processo de cumprimento e execução de tarefas pré-determinadas. Desta forma o ambiente de trabalho deve ser transformado num local de desenvolvimento e evolução.

\section{PROPOSTAS DE AÇÕES DE QUALIDADE EM SERVIÇOS DE SAÚDE}

Serviços de saúde são estabelecimentos que tem como objetivo cuidar de pessoas doentes no sentido de as conduzir de volta ao melhor estado de saúde possível. Neste sentido estes serviços não contemplam unicamente o diagnóstico e tratamento de doenças, mas também se dedicam a desenvolver ações e programas de promoção da saúde e prevenção de doenças. No Brasil, de acordo com a lei (16) "entende-se por serviços de saúde qualquer edificação destinada à prestação de assistência à saúde da população, e todas as ações de promoção, recuperação, assistência, pesquisa e ensino em saúde em qualquer nível de complexidade”.

Tomando como referência a missão-visão de dois grandes complexos de serviços de saúde da capital de São Paulo podemos perceber a preocupação com a QPF perpassando pelos demais elementos apontados acima. Um deles, compactamente, define sua missão-visão como o "orgulho de fazer o melhor para as pessoas, com as pessoas - compromisso e razão pela qual cada membro da instituição dia após dia dedica seus esforços". O outro, por sua vez aborda de forma mais abrangente, declarando ser sua missão "promover o bem-estar físico, psíquico e social do ser humano com profissionais qualificados e tecnologia atualizada, buscando atingir a excelência no atendimento, ensino e pesquisa". Em continuidade explicita a visão do estabelecimento: "ser reconhecida pela excelência no atendimento, ensino, pesquisa e gestão em saúde". Para o atingimento desses objetivos tem como valores: o respeito ao ser humano; ensino e pesquisa; valorização dos colaboradores; excelência profissional; e visão integrada e realista da assistência, entre outras.

Estudando a insatisfação no trabalho de profissionais da saúde, Sorato et al. (2017) (17) demonstram que "os aspectos geradores de insatisfação no trabalho estão relacionados às categoriais: gestão do trabalho em saúde, relações com a equipe e usuários e excesso de trabalho". Afirmam também que "a gestão do trabalho em saúde agregou os seguintes fatores: estrutura física inadequada, falta de recursos materiais, déficit salarial, falta de valorização do trabalho, problemas na gestão e jornada de trabalho excessiva". Chamam a atenção para o fato que "na categoria relações com a equipe e usuários" a violência, falta de conhecimento da Estratégia Saúde da Família, postura do usuário, falta de qualificação da equipe e deficiência na organização do trabalho são determinantes importantes na determinação do nível de insatisfação dos trabalhadores de serviços de saúde. Por fim, na categoria 
"excesso de trabalho" as variáveis mais apontadas são: "sobrecarga de trabalho, excesso de demanda e burocracia".

Desta forma este tema deve ser analisado considerando a "equação da qualidade" proposta.

\section{Qualidade dos recursos disponíveis (QRD)}

É fato que o estresse é um elemento prejudicial ao desenvolvimento do trabalho impactando diretamente na saúde do trabalhador e na qualidade do serviço ofertado e, em decorrência, na satisfação do usuário. Um ambiente físico de trabalho desconfortável, negligente quanto às instalações, equipamentos e recursos necessários para a adequada execução das tarefas, desasseado, ruidoso, mal arejado, mal ventilado e mal iluminado, pouco ou nada ergonômico, etc. configura-se como altamente estressor e deve ser objeto de atenção por parte do gestor e de investimento da organização no sentido de corrigir estas não conformidades.

\section{Qualidade técnico-operacional (QTO)}

Na gestão de serviços de saúde algumas situações devem ser evitadas no ambiente, processo ou método de trabalho objetivando garantir a qualidade:

a) tarefas que exijam sustentação/transporte manual de peso;

b) desequilíbrio entre momentos de atividade e repouso;

c) alongamento das jornadas de trabalho;

d) repressão da iniciativa e da criatividade individual na construção das rotinas de trabalho e desprezo pela participação do trabalhador no processo de construção do modelo de produção e prestação de serviço;

e) exagerada centralização diretiva das decisões sobre a execução das tarefas;

f) ausência de canais de comunicação entre os níveis hierárquicos;

g) falta de política de interação entre o conjunto dos trabalhadores, ambiente de trabalho e vida familiar do trabalhador;

h) modelo de relações interpessoais caracterizado pela falta de atenção para o convívio social dentro da empresa e de intercâmbio de ideias e ajuda na execução do trabalho;

i) modelo de oportunidades caracterizado pela falta de perspectivas claras de ascensão pessoal no emprego e de estímulos de acesso na estrutura empresarial. 
As condições acima expostas caracterizam-se como estressores ocupacionais geradores de uma série de sintomas físicos e emocionais. Além de sintomas emocionais (ansiedade e angustia, por exemplo), estes estressores induzem sintomas comportamentais como a ingestão de bebidas alcoólicas e medicalização.

\section{Qualidade de vida no trabalho (QVT)}

Nos serviços de saúde, os trabalhadores podem estar expostos a uma série de agentes de riscos à saúde, denominados agentes de risco ocupacional. Dentre eles destacam-se os agentes de natureza biológica, a saber: microrganismos, geneticamente modificados ou não; culturas de células; parasitas; toxinas e príons. (16) Estes agentes são capazes de provocar danos à saúde humana por meio de infecções, efeitos tóxicos, alergias, doenças autoimunes, malformações e câncer (18).

Além das agressões decorrentes da exposição desprotegida a agentes biológicos, o trabalhador da área da saúde está exposto também a agentes químicos (gases, vapores, fumos, quimioterápicos etc.), físicos (radiações ionizantes e não ionizantes), biomecânicos (ortostatismo prolongado, sustentação de peso etc.) e psicossociais \& organizacionais (estresse, trabalho em período noturno, imposição de rotina intensa) que também provocam doenças em trabalhadores

Os trabalhadores de serviços de saúde estão ainda expostos a risco de acidentes, sobretudo com material perfurocortante (agulhas com lúmen e sem; laminas, estiletes, etc.), que pode produzir danos à saúde do trabalhador, sobretudo se o material foi utilizado em paciente portador de doença infectocontagiosa (por exemplo: hepatite B ou C, HIV).

Assim, criar no ambiente de trabalho condições saudáveis e seguras focadas na prevenção das doenças e acidentes do trabalho é fundamental e constitui o primeiro passo para a QVT em serviços de saúde.

Em nosso país, as condições mínimas relacionadas para a segurança e saúde no ambiente do trabalho estão previstas em legislação específica denominada Normas Regulamentadoras (NR) (19).

As NR fazem parte de lei federal que regulamenta ações necessárias para a promoção de local seguro de trabalho, sendo de observância obrigatória pelas empresas privadas e públicas que possuam empregados regidos pela Consolidação das Leis do Trabalho (CLT).

Com o objetivo de desenvolvimento de ações de QVT mesmo para empresas com trabalhadores não regidos pela CLT, recomenda-se observação das NR, justificada pela funcionalidade e abrangência desta legislação ao propósito.

Assim, em busca da efetivação das ações de QVT em serviços de saúde recomenda-se, mínima e preliminarmente a observação às NR de números 4 , $5,6,7,9,15,17$ e 32 . 
A NR 4 dispõe sobre os serviços especializados em engenharia de segurança e em medicina do trabalho (SESMT) estabelecendo que este serviço tem a finalidade de promover a saúde e proteger a integridade do trabalhador no local de trabalho.

A NR 5 dispõe sobre a comissão interna de prevenção de acidentes (CIPA) com o objetivo de prevenir acidentes e doenças decorrentes do trabalho, de modo a tornar permanentemente compatível o trabalho com a preservação da vida e a promoção da saúde do trabalhador.

A NR 6 orienta sobre equipamento de proteção individual (EPI); considera-se EPI todo dispositivo ou produto de uso individual utilizado pelo trabalhador destinado à proteção de riscos suscetíveis de ameaçar a segurança e a saúde no trabalho.

A NR 7 regulamenta o programa de controle médico de saúde ocupacional (PCMSO); esta norma estabelece a obrigatoriedade de elaboração e implementação do PCMSO com o objetivo de promoção e preservação da saúde do conjunto dos seus trabalhadores.

A NR 9 estabelece a obrigatoriedade da elaboração e implementação, por parte de todos os empregadores e instituições que admitam trabalhadores como empregados, do programa de prevenção de riscos ambientais (PPRA), visando à preservação da saúde e da integridade dos trabalhadores, através da antecipação, reconhecimento, avaliação e consequente controle da ocorrência de riscos ambientais existentes ou que venham a existir no ambiente de trabalho, tendo em consideração a proteção do meio ambiente e dos recursos naturais.

A NR 15 trata das atividades e operações insalubres fornecendo subsídios técnicos e legais para sua gestão.

A NR 17 dedica-se à ergonomia e visa estabelecer parâmetros que permitam a adaptação das condições de trabalho às características psicofisiológicas dos trabalhadores, de modo a proporcionar o máximo de conforto, segurança e desempenho eficiente.

A NR 32 tem por finalidade estabelecer as diretrizes básicas para a implementação de medidas de proteção à segurança e à saúde dos trabalhadores dos serviços de saúde, bem como daqueles que exercem atividades de promoção e assistência à saúde em geral.

A implantação e desenvolvimento de ações para a promoção de QVT em serviços de saúde devem estar obrigatoriamente relacionados aos resultados observados na realização do PCMSO.

O PCMSO é, desta forma, utilizado como instrumental clínico-epidemiológico que considera os desvios de saúde que incidem sobre os trabalhadores relacionando-os com o trabalho.

O PCMSO é implementado valendo-se de exames médico-ocupacionais, a saber: admissional, periódico, de retorno ao trabalho, de mudança de função e 
demissional. Todos estes exames devem considerar os agentes aos quais os trabalhadores estão expostos e o risco decorrente e observar a eventual ocorrência de doenças advindas do contato com estes agentes.

Na constatação de doença ocupacional, os especialistas do SESMT, valendose de ferramental investigativo clínico e epidemiológico, poderão identificar causa e concomitantes do evento e definir ações corretivas com o objetivo de reduzir, até eliminar, o(s) agente(s) determinantes e, desta forma, garantir um trabalho seguro e saudável.

Para melhorar a eficácia do programa de QVT, além de ações consequentes ao perfil epidemiológico observado no PCMSO, propõe-se o desenvolvimento de programas que incentivem a responsabilidade laboral (cuidado com a salubridade e segurança no trabalho: "espírito prevencionista").

\section{Qualidade de vida geral (QVG)}

Valendo-se da realização dos exames periódicos de todos os trabalhadores, é possível obter, por setores da empresa, a frequência e natureza das doenças prevalentes (perfil de morbidade), sejam estas ocupacionais, ou não, incidentes na força de trabalho. Estes dados são registrados em documento previsto na norma denominado Relatório Anual do PCMSO.

Observando-se os resultados do Relatório Anual, poderá ser proposto o planejamento e desenvolvimento de ações para a promoção, prevenção e proteção às doenças e anormalidades clínicas observadas. Importante registrar que o Ministério da Saúde propõe uma ação nacional para criar políticas públicas que promovam estilos de vida mais saudáveis em todas as etapas da vida, favorecendo a prática de atividades físicas no cotidiano e no lazer, o acesso a alimentos saudáveis e a redução do consumo de tabaco.

Algumas ações e programas podem ser apontados:

a) Programa de controle do peso - A constatação de expressivo número de trabalhadores com sobrepeso merecerá o desenvolvimento de programa desenvolvido pelo serviço de nutrição e dietética para oferecimento de dietas hipocalóricas aos trabalhadores. A instituição poderá oferecer também programas de atividade física para auxiliar no controle de peso.

b) Programa de prevenção para envelhecimento saudável - Baseado no programa do Ministério da Saúde propõe-se a implantação e implementação de programa que promova estilos de vida mais saudáveis para o trabalhador idoso, favorecendo a prática de atividades físicas no cotidiano e no lazer, o acesso a alimentos saudáveis e a redução do consumo de tabaco. Estas questões são a base para o envelhecimento saudável, que signifique também um ganho substancial em qualidade de vida e saúde. Nesse contexto, com o objetivo de produzir material informativo e suporte técnico à mobilização da 
sociedade para a promoção da saúde, a Secretaria de Vigilância em Saúde reproduziu o documento "Envelhecimento Saudável - Uma Política de Saúde" (12) elaborado pela Unidade de Envelhecimento e Curso de Vida da OMS. Baseado neste documento, propõe-se o desenvolvimento de programas focados em aspectos emocionais, incentivo para a atividade física, recomendação para alimentação saudável e incremento ao lazer, entre outros.

c) Programa de promoção da saúde mental - $O$ enfoque dos aspectos emocionais, por meio da disponibilização de apoio psicológico aos trabalhadores de serviços de saúde, pode ser importante ferramenta para o controle dos agentes de natureza psicossocial e organizacional permitindo a prevenção dos transtornos mentais (20).

d) Programa de promoção de prática de hábitos saudáveis de vida (atividade física regular, alimentação saudável e lazer) - Torna-se necessário também despertar a consciência para a participação dos trabalhadores de serviços de saúde em atividades físicas regulares. Importante divulgar que esta atividade pode retardar declínios funcionais, além de diminuir o aparecimento de doenças crônicas. Enfatizar que uma atividade física regular e moderada reduz o risco de morte por problemas cardíacos em 20 a $25 \%$ em pessoas com cardiopatia diagnosticada. Também pode reduzir substancialmente a gravidade de deficiências associadas às doenças cardíacas e outras doenças crônicas (20). A conscientização da alimentação saudável (20) enfocando o consumo correto de calorias é muito importante para a profilaxia de doenças crônicas. Dietas com excesso de gordura saturada e sal, pobres em frutas, legumes, verduras, fibras e vitaminas são fatores predisponentes para patologias como diabetes, doenças cardiovasculares, hipertensão arterial, artrites e alguns tipos de câncer. O lazer (20), através de programas privilegiando atividades artísticas e culturais, pode atuar como ferramentas de profilaxia aos riscos associados a agente psicossocial e organizacional e fortalecimento do espirito de equipe dos trabalhadores de uma instituição. Atividades como concursos para revelação de talentos existentes na empresa tem demonstrado importantes resultados na melhoria do clima organizacional.

e) Programa de apoio para abandono do hábito de fumar - Ação como o incentivo ao abandono do cigarro (20) pode ser importante instrumento para a qualidade de vida do trabalhador considerando-se que 0 tabagismo é fator de risco de doença não transmissível modificável e representa a causa de morte prematura mais evitável. Importante conscientizar o trabalhador de que deixar de fumar representa a diminuição do risco de câncer de pulmão, da perda funcional, prevenindo ainda malefícios como diminuição da densidade óssea, da força muscular e da função respiratória. A empresa necessitará assumir que os custos decorrentes para a efetivação do abandono do fumo se trata de um investimento, cujo retorno ocorrerá pela melhoria da saúde do trabalhador. 
f) Programa de apoio para abandono do uso de álcool - Programas para alcoolistas (20) podem representar importante auxilio para trabalhadores que individualmente não conseguem controle da ingestão etílica. Registre-se que o alcoolismo impacta agudamente no absenteísmo, devido aos efeitos proporcionados pelo consumo. Este vício impacta também ao longo do tempo envolvendo doenças relacionadas ao álcool, como desnutrição e doenças do pâncreas, estômago e fígado.

g) Programa de educação financeira - Programas de educação financeira (20) podem ter valia para os trabalhadores de serviços de saúde no subsidio de planejamento de patrimônio e reserva econômico-financeira futura e para após a aposentadoria.

h) Programa de preparação para a aposentadoria - Programa de preparo para o período de cessação da atividade laboral (20) pode ter importante impacto junto aos trabalhadores pela projeção de ideia de conscientização para a importância da preservação da saúde física e mental na aposentadoria.

i) Programa de apoio à mãe trabalhadora - Projetos como creches junto ao ambiente de trabalho podem constituir diferencial na fidelização de servidores, além de proporcionar tranquilidade aos pais que podem contar com filhos próximos ao ambiente de trabalho.

Finalmente, deve ser salientado que para a consecução da QVG, além de ações consequentes ao perfil epidemiológico observado no Relatório Anual, propõe-se o desenvolvimento de programas que incentivem a responsabilidade pessoal (cuidado consigo mesmo ou autocuidado).

\section{DISCUSSÃO}

O SESMT é o setor que coordena o desenvolvimento dos programas de QVT e tem papel importante e fundamental nos programas de QVG. Este serviço especializado, além de profissionais previstos na NR 4, deve incorporar outros profissionais como psicólogos, assistentes sociais, educadores físicos e outros especialistas passiveis de contribuir para o bem-estar e a melhoria da QVT.

No desenvolvimento dos programas para o bem-estar, o SESMT utiliza indicadores de desempenho para monitoramento dos resultados. A efetividade dos programas será observada por indicadores como queda do número de acidentes do trabalho e de doenças ocupacionais com consequente redução do absenteísmo, aumento da produtividade e melhora do clima organizacional.

O SESMT, ao monitorar estes indicadores, deve realizar análises comparativas dos resultados desejados e dos resultados observados. Na observação de resultados aquém do esperado tornam-se necessárias ações de correção para a consecução do desejado. A aplicação de uma metodologia administrativa 
como o ciclo PDCA (planejar, executar, controlar e atuar - sigla em inglês) é fundamental para a gestão das ações.

O administrador do SESMT, responsável pelos programas de QVT não deve aguardar que a simples implantação de ações e programas para o bem-estar no trabalho proporcionem imediatamente a melhoria da QV dos trabalhadores.

A melhoria do clima organizacional, diminuição do absenteísmo, queda do número de doenças relacionadas ao trabalho ocorrerão na medida direta de quanto o trabalhador contribuir com adoção de estilo de vida saudável e participação ativa nos cuidados à saúde.

Na caminhada em busca da QV dos trabalhadores de serviços de saúde, o SESMT necessitará contar com o apoio continuo das comissões de controle de infecção hospitalar para o desenvolvimento dos programas de QVT, considerando-se que os agentes biológicos são determinantes importantes para a ocorrência de doenças em trabalhadores de serviços de saúde.

Parcerias importantes do SESMT para a consecução de programas de QVT são também as efetivadas com comissões representativas dos trabalhadores, exemplificadas pela CIPA e pela comissão de saúde do trabalhador (COMSAT), no caso dos hospitais públicos do estado de São Paulo, que podem contribuir com sugestões para a melhoria da segurança e da saúde nos ambientes de trabalho. Estas comissões representativas dos trabalhadores têm papel fundamental para a QVT já que de nada adiantará oferecer segurança e saúde no trabalho, caso não exista a conscientização de que há necessidade de o trabalhador contribuir com sua parte: usar os equipamentos de proteção, realizar exames periódicos, precaver-se com as possibilidades de acidentes, enfim ter cuidado consigo.

\section{CONCLUSÃO}

Os resultados das ações da gestão de pessoas no eixo da QVT podem proporcionar aos serviços de saúde resultados como diminuição de acidentes de trabalho, decréscimo de doenças ocupacionais, melhoria do clima organizacional e consequente aumento da produtividade.

Ideal seria que a simples implantação de programas para a prevenção e promoção à saúde pudessem proporcionar o bem-estar e melhoria da qualidade de vida dos trabalhadores. Porém, para este objetivo são necessárias mudanças de paradigmas. É fundamental fazer com que o trabalhador compreenda a importância das medidas de prevenção, como o uso correto e rotineiro dos equipamentos de proteção, da necessidade de monitoramento de sua saúde e da importância do autocuidado. O administrador dos serviços de saúde precisa conscientizar-se de que o bem-estar e a qualidade de vida dos trabalhadores de serviços de saúde são alicerces da qualidade assistencial, pois somente encontrando-se saudável e satisfeito, o profissional poderá contribuir para a melhor assistência do paciente e da comunidade. 


\section{REFERÊNCIAS}

1. Brian, E; Becker, DU; Huselid, MA. Gestão Estratégica de Pessoas com Scorecard. Ed. Elsevier, Brasil, 2001.

2. Burmester H; Aidar MM. Planejamento Estratégico e Competitividade em Saúde. Ed. Saraiva, Brasil, 2017.

3. WHO. The World Health Organization quality of life assessment (WHOQOL) The WHOQOL Group 1995. Position paper from the World Health Organization. Social Science and Medicine 10:1403-1409. Disponível em https://www.ncbi.nlm.nih.gov/pubmed/8560308. Acessado em 14/01/2019.

4. Rodrigues, MVC. - Qualidade de vida no trabalho: evolução e análise no nível gerencial. UNIFOR, Fortaleza, 1991.

5. Walton, RE. Quality of Working Life: What is it? Sloan Management, 15(1): 11- 21,1973.

6. Fernandes, EC. Qualidade de vida no trabalho: como medir para melhorar. Salvador: Casa da Qualidade, 1996.

7. Dantas, J. Coração e fatores de risco. Qualidade total na promoção da Saúde. 1a. ed. Belo Horizonte: Editora o Lutador, 1996. v. 1. 168p.

8. França, ACL. Indicadores empresariais de qualidade de vida no trabalho esforço empresarial e satisfação dos empregados no ambiente de manufaturas com certificação ISO 9000. Tese de Doutorado em Administração - USP,1996.

9. França, ACL. Qualidade de vida no trabalho: conceitos, abordagens, inovações e desafios nas empresas brasileiras. Vol. 1. Rio de Janeiro: [s.n.], 1997.

10. Albuquerque, LG; Limongi-França, AC. Estratégia de Recursos Humanos e Gestão de Qualidade de Vida no Trabalho: O stress e a expansão do conceito de qualidade total. Curso Avançado de Gestão Empresarial em Qualidade de Vida: FEA/USP, São Paulo, 2003. Disponível também na Revista de Administração São Paulo, v. 33, no 2, p.40-51, abr - jun de 1998.

11. Limongi-França, AC e Zaima G. Gestão de Qualidade de Vida no Trabalho - GQVT. In: Manual de Gestão de pessoas e equipes: estratégias e tendências. São Paulo: Gente, 2002.

12. Chiavenato, I; Matos, FG. Visão e Ação Estratégica. São Paulo: Prentice Hall, 2002.

13. Maslow, A. H. A theory of human motivation. Psychological Review, 50: 390-6,1943). 
14. Herzberg, Frederick; Mausner, Bernard; Snyderman, Barbara B. The Motivation to Work (2nd ed.). New York: John Wiley and Sons. 1959.

15. Herzberg, Frederick. "One More Time: How Do You Motivate Employees?". Harvard Business Review. 65, September - October 1987.

16. Brasil. Ministério do Trabalho. Portaria GM n $n^{0 .} 485$ de 11 de novembro de 2005. D.O.U. de 16/11/2005. NR 32 - segurança e saúde no trabalho em serviços de saúde. Brasília, 2005).

17. Sorato et all. Insatisfação no trabalho de profissionais da saúde na estratégia saúde da família. Texto Contexto Enferm. 26(3), 2017.

18. Risco Biológico Guia Técnico. Disponível em https://www.unifesp.br/reitoria/dga/images/legislacao/biosseg/guia tecnico cs3. pdf . Acessado em 14/01/2019.

19. Normas Regulamentadoras. Disponível em http://www.tst.jus.br/web/trabalhoseguro/normas. Acessado em 14/01/2019.

20. WHO. WORLD HEALTH ORGANIZATION. Envelhecimento ativo: uma política de saúde / World Health Organization; tradução Suzana Gontijo. Brasília: Organização Pan-Americana da Saúde, 2005. 60p. Disponível em: http://bvsms.saude.gov.br/bvs/publicacoes/envelhecimento ativo.pdf Acessado em 14/01/2019

Recebido: 15 de janeiro de 2019. Aceito: 05 de fevereiro de 2019

Correspondência: Milton M. Osaki. Av. Dr. Enéas Carvalho de Aguiar, 188 Cerqueira César, São Paulo - SP, 05403-000. E-mail: mosaki@saude.sp.gov.br

Conflito de Interesses: os autores declararam não haver conflito de interesses.

(C) This is an Open Access article distributed under the terms of the Creative Commons Attribution License, which permits unrestricted use, distribution, and reproduction in any medium, provided the original work is properly cited 